Estudios de la Paz y el Conflicto

Revista Latinoamericana

IUDPAS-UNAH

Volumen 1, Número Especial, pp. 111-119

ISSN-e: 2707-8922 / ISSN: 2707-8914

DOI: $10.5377 /$ rlpc.v0i0.9507

Anual, 2019

\title{
DESDE LA SORORIDAD, LAS MUJERES RESURGIMOS DE LA GUERRA
}

\author{
FROM SORORITY, WOMEN RISE FROM WAR
}

\author{
Dennis Dussán Márquez \\ Universidad de la Amazonia \\ dennisdussan@yahoo.es \\ Luisa Fernanda Toro Cruz \\ Universidad de la Amazonia \\ lufertoro17@hotmail.com
}

Cómo citar / citation

Dussán, D., Toro, L.F. (2019). "Desde la sororidad, las mujeres resurgimos de la guerra", Estudios de la Paz y el Conflicto, Revista Latinoamericana, Número Especial 1, 111-119. DOI: $10.5377 /$ rlpc.v0i0.9507

\section{Resumen}

En el Departamento del Caquetá, Colombia, el conflicto armado tuvo su máxima expresión, su mayor y dolorosa presencia, afectando la población en todos sus municipios y veredas. Ha sido en este trascurso de tiempo, desde la firma de los acuerdos de Paz entre el Gobierno Nacional y las Fuerzas Armadas Revolucionarias de Colombia (FARC), que los investigadores regionales, en particular los jóvenes investigadores, nos hemos dado a la tarea de acercarnos a una realidad que antes, por la misma barbarie del conflicto y su asentamiento territorial, no habíamos logrado hacerlo. La realidad de la guerra y sus efectos en las poblaciones ha sido una problemática principal asumida por nuestro semillero Inti Wayra y del grupo de investigación Mandala de Pensamiento, en donde nos hemos congregado jóvenes y profesores, afectados por el conflicto armado, rescatando nuestros testimonios para acercarnos a la comprensión de lo sucedido al menos durante los últimos 20 años. Este artículo presentará las formas en que las mujeres víctimas de violencia sexual y otros tipos de violencia han resurgido de la guerra a través de la sororidad.

\section{Palabras claves}

Mujeres; Sororidad; Guerra; Paz. 


\begin{abstract}
In the Department of Caquetá, Colombia, the armed conflict had its maximum expression, its greatest and most painful presence, affecting the population in all its municipalities and villages. Since the signing of the peace agreement between the National Government and the Revolutionary Armed Forces of Colombia (FARC), regional researchers, particularly, young researchers have been approaching a reality that before, due to the barbarity of the conflict and its territorial settlement, had not been able to do it. The war and its effects on populations have been a major subject of analysis for our research groups; Inti Wayra and Mandala de Pensamiento. We are a group of students, teachers, and researchers affected by the armed conflict who are rescuing our testimonies to understand what happened in our region for the last 20 years. This article presents how women have overcome sexual violence and other forms of violence through sisterhood and solidarity.
\end{abstract}

\title{
Palabras clave
}

Women; Sorority; War; Peace.

\section{INTRODUCCIÓN}

Queremos reseñar inicialmente en el presente artículo de la manera en cómo ha sido la participación de los jóvenes investigadores con el acompañamiento de profesores, en torno a las relaciones construidas por las mujeres víctimas de violencia sexual, que en su manera de nombrar nos han presentado la palabra "sororidad", a la cual han dado un gran significado, como corazones que se hermanan, almas que se hermanan e historias que se hermanan, sororidad presente en los encuentros fraternos, que nacen del amor y la compasión, del no juzgar, del escuchar y del ayudar.

Escuchando a María Argensola Quintero ${ }^{1}$ quien es una mujer lideresa, víctima de violencia sexual en el marco del conflicto armado, integrada a nuestro semillero de investigación, quien nos habla de este significado de la "sororidad", desde la voz de una persona renaciente como es ella:

"La palabra sororidad o sorora es quien se ha comprometido con las otras personas y aún más, esta palabra se aplica mucho para nosotras las mujeres; nosotras las mujeres tenemos un gran problema y es que nos agredimos demasiado, no tenemos esa capacidad de entendimiento y de ponernos los zapatos de la otra, esos zapatos que nos pueden quedar apretados, grandes o de pronto hasta pequeños, por el contrario siempre estamos juzgando a la otra, y esta, es la palabra que nos da apertura al reconocimiento de la solidaridad, de esa solidaridad con la otra, de cómo siento yo a la otra desde el punto de vista de una agresión, de una violencia sexual, de una dificultad que tenga mi otra compañera, pero lo que se presenta en estos casos es que inmediatamente entramos es a juzgar.

Ese juzgamiento "a esa la violaron porque eran las dos de la mañana, estaba en la calle y además de eso estaba casi semi desnuda", resulta que casi todas las mujeres vivimos con mucha represión,

\footnotetext{
${ }^{1}$ Diálogo en torno a la palabra sororidad con la lideresa de la comunidad indígena embera chamí, María Argensola Quintero Bañol, quien es integrante del semillero Inti Wayra. Estos diálogos se realizan entre estudiantes, profesores y líderes - lideresas, a manera de círculos de reflexión, para lograr dar significado a conceptos que se utilizan, en este caso en los grupos de mujeres, teniendo como referencia el contexto histórico y territorial, que nos permita dar una resignificación a este concepto sororidad. Estos diálogos se realizaron en el transcurso del 66año 2019 en la Universidad de la Amazonia.

Estudios de la Paz y el Conflicto, Revista Latinoamericana, IUDPAS-UNAH, Número Especial 1, 2019, pp. 111-119.

DOI: $10.5377 /$ rlpc.v0i0.9507
} 
el machismo nuestro gran enemigo que marca nuestro sufrimiento, ese machismo es el que tenemos que combatir y de qué manera lo podemos hacer, solo si nosotras las mujeres somos sororas entre sí, de estar siempre con la otra, de esa mujer que está siendo agredida, que está siendo violentada, que está siendo vulnerada o que está siendo afectada".

Fuimos comprendiendo este importante significado, que sería la clave para lograr una buena articulación con las mujeres, la creación de un buen escenario de dialogo que acercara a las mujeres universitarias víctimas del conflicto con las mujeres lideresas comunitarias y del movimiento social, estando en igualdad de condiciones, ya que veníamos de historias similares, que no son otra cosa que historias de las supervivientes.

El semillero Inti Wayra se enfocó con uno de sus grupos en esta investigación, sobre la problemática de las mujeres víctimas de violencia sexual, para acercarnos a la comprensión de una triste y dolorosa realidad, realidad silenciada, historia silenciada, memoria silenciada, por el horror de cada una de las experiencias de las mujeres y bajo este manto de silencio, era imposible avanzar en una mayor comprensión de la problemática que se quería abordar.

En la Universidad de la Amazonia, ya habíamos tenido experiencias a través de diferentes cátedras de sociología y metodología de la investigación, con preguntas emergentes acerca de la realidad que los jóvenes universitarios podíamos proponer como problemas de investigación y al acercarnos a la realidad del conflicto armado, desde la propuesta de explorar en torno a las propias experiencias de cada uno de nosotros, nos topábamos con un muro casi infranqueable de silencio, que nos llevaba la negación de estar inmersos o ser parte de la problemática que se quería abordar.

Fuimos parte dialogante de un proceso de investigación llevado a cabo por la Docente Dennis Dussán Márquez, doctorante del Instituto de Pensamiento y Cultura para América Latina de México (en adelante, IPECAL), en el cual acogíamos una metodología que acoge en el proceso de conocer a manera de una matriz epistémica al sujeto y su subjetividad, a su experiencia, a su realidad, a su historia, al contexto mismo en donde el sujeto vive y pervive, y desde allí con sus emociones y experiencias a flor de piel, construye el campo problemático de su investigación. Esta fue una metodología muy potente, la que nos presentaba desde la voz de la profesora a los pensadores latinoamericanos Hugo Zemelman y Estela Quintar ${ }^{2}$, en el liderazgo de las nuevas corrientes latinoamericanas conocidas como "epistemologías del sur” en su denominación nombradas por Boaventura de Soussa Santos (2018).

A partir de este proceso fuimos plasmando y acotando una metodología que pudiera cobijarnos en las subjetividades que se construyen en el conflicto armado, en los efectos que sufren sociedades que han vivido de manera constante y permanente la crueldad de la guerra, que para el caso colombiano, data de más de 70 años sin respiro ni tregua. ¿Cómo nos formamos como sociedad y como sujetos, aquellos que vivimos bajo el yugo de la opresión, la muerte y la tortura, la desaparición forzada, la violencia sexual, de una generación a otra?

\footnotetext{
${ }^{2}$ Hugo Zemelman (2002) y Estela Quintar (2006) son precursores de metodologías y didácticas de construcción de pensamiento crítico, teniendo como referente el pensar latinoamericano, desde un cuestionamiento al eurocentrismo como eje en la formación de investigadores latinoamericanos; lo que nos determina una imposición de la teoría ante las prácticas y experiencias del sujeto que se "historiza", recupera su realidad y se potencia en su subjetividad, en pos de la transformación de esa realidad, que como hemos visto es una realidad abrumadora, bestial, deshumanizante. Finalmente es una apuesta por humanizar los procesos académicos y sociales para crear conciencia de la necesidad de transformación y recuperación del sujeto para la vida y de la vida en el sujeto que investiga.
}

Estudios de la Paz y el Conflicto, Revista Latinoamericana, IUDPAS-UNAH, Número Especial 1, 2019, pp. 111-119. DOI: $10.5377 /$ rlpc.v0i0.9507 
Desde los espacios dialogantes, también como aprendizaje de IPECAL, fuimos transformando nuestros espacios pedagógicos de los círculos de reflexión, en amplios círculos de palabra y fuimos también nombrando las prácticas emergentes como "metodología de la memoria encriptada", como una propuesta que hace frente a la desmemoria; pues, cuando los recuerdos se guardan (se encriptan) y se olvidan o parecen olvidarse. Esta metodología nace para retar al olvido que nace del miedo a sentir dolor por el recuerdo de una muerte violenta y en general de los hechos violentos, miedo que como guardián impide la enunciación del testimonio.

Esta metodología se puso a prueba con la experiencia de investigación con las mujeres víctimas de violencia sexual, en donde nos encontramos con la gran dificultad de su memoria encriptada, traducida al silencio sobre lo ocurrido. Fue así como durante el primer año nos enfocamos en desencriptar la memoria y de esta manera logramos conocer a profundidad lo ocurrido con las mujeres en el Caquetá, durante el conflicto armado. Estos resultados fueron contenidos en la exposición realizada en el marco del XI Congreso del Consejo Latinoamericano de Investigación para la Paz (en adelante, CLAIP) y II Encuentro de la Red de Jóvenes Constructores de Paz, realizado en Sao Paulo, Brasil, en el año 2019.

Para tener un contexto más ilustrativo, vamos a continuación a contar un poco más de cómo se vivió esta guerra en nuestra región:

El departamento del Caquetá, es una de las zonas con mayor afectación por el conflicto armado. Se ubica en la región amazónica colombiana, al sur del país. El proyecto de asistencia social y atención humanitaria inmediata a población víctima del conflicto armado en el municipio de Florencia establece que este municipio es uno de los mayores receptores de población desplazada y otros hechos victimizantes del país con 87.030 víctimas registradas. Estas personas han sido afectadas principalmente por desplazamiento, asesinatos, desapariciones forzadas (11.500), violencia sexual, secuestro, tortura, mutilaciones por minas, entre otros.

Y es también en este vasto territorio en donde los actores de la guerra, en una disputa a muerte por el territorio ocasionaron miles de estas víctimas que hoy se concentran en su mayoría en Florencia, ya sin tierra, sin bienes materiales para subsistir y exigiendo del estado una reparación por el daño ocasionado.

También ha sido un territorio de generosidad y aprendizaje de cómo se puede transformar un imposible, de ser un territorio de guerra "sin cuartel", a un territorio en donde hoy es posible tejer la esperanza, esperanza re-nacida en el año 2016 a partir de la firma de los acuerdos de paz, aunque tendríamos que decir que la guerra es como una enfermedad incurable, ya que a hoy, al año 2020 y desde el año 2018 y 2019, las disidencias de las FARC recrudecen el conflicto, como también organizaciones ligadas al narcotráfico y a la disputa de tierras, que aniquilan la vida de líderes campesinos e indígenas que habitan estos territorios. Todo esto, ante un estado que niega esta realidad, que no actúa, no existe, no persigue, no castiga, no investiga los múltiples crímenes contra los líderes sociales y los liderazgos políticos de los nuevos procesos de paz (solo en 2020, en el mes de enero ha sido asesinados 27 líderes sociales y 4 excombatientes de FARC). Aun así, es un territorio en

\footnotetext{
${ }^{3}$ Se enuncia esta metodología de la "memoria encriptada" a partir de los trabajos de investigación doctoral en el Ipecal-México, de la docente Dennis Dussán Márquez (2018), en donde explora desde su propia experiencia como mujer excombatiente de la insurgencia, esta afectación como "miedo a sentir dolor por el recuerdo de una muerte violenta", cuyos contenidos se expresan en su tesis doctoral "El Poder del Miedo en la Reconfiguración de la Utopía".

Estudios de la Paz y el Conflicto, Revista Latinoamericana, IUDPAS-UNAH, Número Especial 1, 2019, pp. 111-119.

DOI: $10.5377 /$ rlpc.v0i0.9507
} 
donde aún y, a pesar de todas las circunstancias seguimos apostándole a la paz y en particular las mujeres, desde la sororidad.

Imagen 1. Mapa político del Departamento de Caquetá

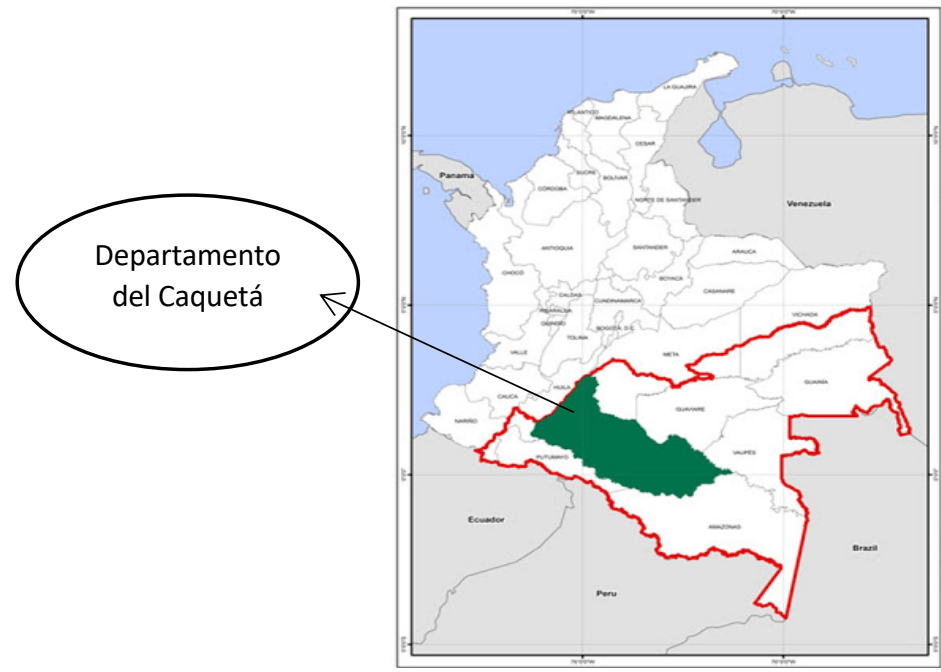

Fuente: http://siatac.co/Atlas/caqueta.html

Aquí, en este territorio es donde está ubicada la Universidad de la Amazonia. Y en este espacio académico y de vida es donde nos hemos congregado, por un propósito importante, como es el poder reconstruir la memoria histórica de las víctimas que sufrieron lo indecible en el marco de la guerra que por más de 50 años afectó nuestro territorio.

Hemos identificado como grupo de investigación y semillero, la barbarie ocasionada en el cuerpo de las mujeres, sobretodo nuestras mujeres campesinas que habitaron los territorios rurales de nuestra región amazónica, región que pasó a ser un sitio estratégico para el accionar de los grupos armados tanto estatales, paramilitares y de la insurgencia.

Desde los encuentros con estudiantes en la reconstrucción e intercambio de las experiencias metodológicas, damos cuenta, cómo a partir de ahí, los jóvenes encontramos la articulación de la investigación en nuestras experiencias en la recuperación del sujeto y su subjetividad: en nuestro caso desde esta recuperación nos enfocamos en la investigación de mujeres víctimas de violencia sexual en el marco del conflicto armado en el Departamento del Caquetá. A partir de este trabajo de investigación, podemos exponer cómo las mujeres hemos logrado recuperar la subjetividad a través de experiencias de vida y cómo esto nos ha llevado a tejer lazos desde la sororidad teniendo en cuenta que estos encuentros nos llevan a construir nuevos conocimientos, que generan espacios de reflexión, paz, perdón y reconciliación desde los escenarios vividos en la guerra.

\section{LA METODOLOGÍA DE LA MEMORIA ENCRIPTADA}

El primer elemento de esta metodología, a manera de un potente dispositivo didáctico, que da apertura a la reflexión y se mantiene durante todo el proceso, es la "Mandala de la Esperanza": con jóvenes estudiantes provenientes de diversos pueblos indígenas y mujeres lideresas, fuimos incorporando a nuestros círculos de reflexión una nueva manera de acercarnos a este mundo del conocer, que implicaba pasar por el dolor al contar nuestras 
historias en el conflicto, fue realmente un importante descubrimiento el que nos donaban, ya que al hacer los círculos de palabra integrando lo ritualístico de pedir permiso a los ancestros para traer sus historias al presente, para de esta manera comprender las lecciones de vida que con su muerte nos dejan, ritualístico en poder integrar el poder del agua, el fuego, la tierra y el aire, para generar una energía de conexión espiritual entre nosotros seres humanos y con la naturaleza como parte de todo nuestro universo, ritualístico en el hecho de agradecer la presencia por el hecho de ser supervivientes y poder dar testimonio de lo ocurrido, ritualístico que nos permitió reconocer que somos todos parte de una misma historia.

Estas mandalas iniciaron en la Universidad con los jóvenes investigadores que estaban ya en la disposición metodológica anteponiendo la pregunta: ¿cómo cada uno vivió la guerra en el Caquetá?, pregunta que da recuperación de los relatos de los jóvenes y sus vivencias. Y fue allí donde emergió uno de los primeros relatos de la violencia sexual, en el marco del conflicto armado:

"Historia muy cercana a mis lazos afectivos, me cuenta ella, tan distante, tan amorosa, tan humana, ella con su mirada perdida, sin hacer conexión alguna a la mía, me cuenta el aire de su voz tenue, un aire lleno de melancolía, dolor y algo lejano de esperanza, las atrocidades que vivió en las selvas verdes del departamento del Caquetá, selva que se convirtió en testigo mudo de los hechos macabros de una venganza librada por la amante de su marido, quien sin compasión alguna ordenó a sus hombres que la secuestraran e hiciera con ella lo que ya sabían. Vegetación selvática, arbustos y aguas cristalinas era lo único que podía mirar en el trayecto al lugar de la barbarie, el dolor, la desolación y la impotencia. En ese lugar manos ásperas recorrieron su cuerpo, sin lograr comprender el motivo de su elección; pasó la noche y su cuerpo era rotado como una botella en una mesa de cantina, cinco hombres destrozaron su cuerpo, sus esperanzas y sus sueños. Me cuenta de manera animada, su plan para poder huir de ahi, sin fuerza alguna, con su cuerpo desvanecido, pero con la mirada de sus hijos en sus pensamientos y el recuerdo de su voz diciendo "Mamá", eso la llenaba de esperanza y amor maternal. Esperó a que sus agresores se durmieran y como sabian ellos lo impotente y desgastada que había quedado en el suelo, no le dieron importancia alguna, sigilosa sale del espacio donde fue agredida hasta encontrar el gris lejano de una carretera que le devuelve la esperanza del regreso a casa, corre por la vía con sus prendas desgastadas, rasgadas y embarradas, intentando llamar la atención de alguien, "muchos carros pasaban y al mirarme más aceleraban" me menciona cerrando sus ojos e inclinando su cabeza, pero muy lejano una volqueta venía y de repente vio como las luces de las estacionarias se encienden, abren la puerta y la invita a subir, en el camino solo lloraba; el conductor un hombre gordo, con barba y con olor penetrante a sudor, solo la miraba y se cuestionaba sobre su estado... es así como pude regresar a casa menciona mientras una lagrima baja por su rostro... ${ }^{4}$

Desde la recuperación de este relato y desde su propio reconocimiento como afectada como mujer en el conflicto armado, también por el hecho de haber sido su padre asesinado en Pitalito, Huila, surge la pregunta, si más mujeres pudieron sufrir esta violencia más allá de la universidad y a partir de allí fuimos encontramos relatos en donde la violencia sexual era otro más de los hechos victimizantes vividos por mujeres en el marco del conflicto armado en el Caquetá, encontrando también el relato de la estudiante Natalia Marroquín y la líder social María Argensola Quintero, y luego ampliamos el espacio de la reflexión

\footnotetext{
${ }^{4}$ Relato construido por Luisa Fernanda Toro Cruz, a partir de los círculos de palabra en la cátedra de Sociología Jurídica, orientada por la docente Dennis Dussán Márquez. Universidad de la Amazonia, año 2017.
} 
universitaria a espacios con otras mujeres lideresas, para poder reconocer como estas mujeres pueden recuperar sus historias y ser mujeres renacientes del conflicto armado colombiano.

\section{PRESENCIA LATINOAMERICANA}

Estas voces empezaron a consolidarse y a salir con mucha más fuerza, a través de diversos dispositivos didácticos, los cuales además de la mandala de la esperanza, la reconstrucción del relato de la propia historia, las terapias del agua, florales y de plantas medicinales, los tejidos, entre otras, lograron emerger las voces de resistencia, salidas del dolor y la vergüenza, para erigirse en voces de dignidad y lucha. Estas voces se hicieron presentes en escenarios nacionales como: el Encuentro con Grupos Regionales de Memoria Histórica, desarrollado en la ciudad de Cali por dos años consecutivos, en los Agostos Latinoamericanos, desarrollados por el Instituto IPECAL, que se llevó acabo en la ciudad de Medellín, en diferentes municipios del departamento del Caquetá, en campamentos de semilleros y colectivos de investigación y en escenarios como ponentes en la universidad de la Amazonia, en España en el marco del Huqagamichelwano y al festival de música what is music, desarrollado por el colectivo Memoria Viva, en México en los Octubres Latino Americanos desarrollado por el IPECAL y en la Universidad Nacional Autónoma de México (en adelante, UNAM) en el seminario Internacional de Justicia Transicional.

Este impacto nacional logró generar vínculos a nivel Internacional, con la participación de Luisa Fernanda Toro Cruz, al XI Congreso del Consejo Latinoamericano de Investigación para la Paz y el II Encuentro de la Red de Jóvenes Constructores de Paz: Encuentro mágico, lleno de unión, fraternidad, de espacios de reflexión, palabra, cooperación, esperanza, sororidad y amor, en medio de la música, la palabra, el encuentro, el contacto y la mandala. El encuentro con otras mujeres se empezó a tejer en Brasil, para luego conocer de las otras experiencias con el trabajo que hacen las jóvenes en la UNAM frente a la violencia sexual en esa Universidad y proyectando ahora en Colombia la construcción de nuestro nodo de Jóvenes Latinoamericanos por la No Violencia, en el marco de la programación del II Campamento Juvenil de Semilleros y Colectivos de Investigación a realizarse en la Universidad de Caldas, Manizales, el próximo agosto 2020.

\section{CONCLUSIÓN}

La vida de jóvenes y docentes pasa por la mandala y con la mandala pudimos compartir con jóvenes de Latinoamérica nuestras vivencias y experiencias en la construcción de paz y aperturar nuevas preguntas que son las que nos guían hacia los nuevas conceptualizaciones, encuentros y construcciones como jóvenes y como docentes en nuestros centros universitarios y con las comunidades: ¿Cómo construyo paz desde diferentes metodologías que me acerquen a las realidades de mi país y Latinoamérica?, ¿cómo desde la sororidad de las mujeres nos fortalecemos en resistencia, para reivindicar los derechos arrebatados?, ¿cómo y de qué manera una postura desde las jóvenes y lideresas en clave de resistencia no violenta, puede emerger cuando se ha naturalizado la violencia en la construcción de la propia cotidianidad?

Los espacios de formación e investigación construidos desde el grupo de Investigación y del Semillero, al poner de presente y al centro al sujeto y su subjetividad, a partir de la recuperación de sus propias historias, teniendo el contexto del conflicto y la construcción de 
paz, viene permitiendo desentrañar las voces en un territorio silenciado por el miedo, ya que la barbarie y el terror se impusieron como conducta social, obligando de esta manera al desarraigo y permitiendo el despojo de millones de familias en Colombia (7'816.500 de personas han sido registradas en la Unidad de Víctimas de Colombia, como desplazadas de sus territorios) (Acnur, 2019).

Este despojo, para el caso de las mujeres en el Caquetá, pudo darse, en la medida en que eran reducidas totalmente en su capacidad de reacción, una vez asesinaban a sus compañeros e hijos, o los desaparecían o mutilaban, luego ellas, sufrían la más aberrante violencia sexual, para después obligarlas a abandonar sus tierras. También fueron asesinadas quienes se atrevieron a liderar procesos de reclamación de derechos.

Las mujeres, tímidamente empiezan a levantarse, a enfrentar el miedo y la vergüenza, a liberarse del sentimiento de culpa que las persigue, atreviéndose a desencriptar su memoria del olvido, para dignificarse y erigir su testimonio como una afrenta a la maldad y la injusticia que han vivido, pero por, sobre todo, para desentrañar realmente lo que estuvo detrás de esta guerra y cuál fue el papel del estado y sectores de la sociedad en este despojo.

Ellas ahora, cuentan con un espacio importante como lo es la Comisión de la Verdad, creada a partir de los acuerdos de paz suscritos entre el Gobierno Nacional y las FARC en el año 2016, al cual podrán acudir a plasmar sus testimonios para que esta tragedia se reconozca por parte del estado y los actores armados. ¿Siendo el estado, constitucionalmente garante de los derechos humanos de los colombianos y colombianas, porque no garantizó el derecho a la vida e integridad de las mujeres caqueteñas? ¿Reconocerán los actores armados la vulneración ocasionada en el marco del conflicto armado, a las mujeres caqueteñas y se logrará de una $\mathrm{u}$ otra manera acceder a una reparación digna y a un compromiso de no repetición? Son preguntas que nacen de este nuevo ejercicio ante la Comisión de la Verdad.

Las preguntas que emergen, viene posibilitando el aprendizaje, la reflexión y el intercambio de vivencias en la construcción de paz, en espacios que generan la reconstrucción de nuestra historia a partir de lograr recuperar la subjetividad, generando también y principalmente, un espacio de sanación y una conexión importante para comprender la praxis sentipensante ${ }^{5}$ de jóvenes investigadores y comunidades con quienes se comparte la vida.

\section{REFERENCIAS}

Acnur (Agencia de las Naciones Unidas para los Refugiados) (2019). Informe Tendencias globales: desplazamiento forzado en 2018. (Disponible online: https://www.acnur.org/stats/globaltrends/5d09c37c4/tendencias-globales-dedesplazamiento-forzado-en-2018.html).

Dussán, D. (2018). El Poder del Miedo en la Reconfiguración de la Utopía. Tesis Doctoral. Ipecal, México.

\footnotetext{
${ }^{5}$ El concepto sentipensante es incorporado en las reflexiones del sociólogo colombiano Orlando Fals Borda, el cual, menciona, es recuperado de diálogos con pescadores del Municipio San Benito Abad, Departamento de Sucre, Colombia: "actuar con el corazón y emplear la cabeza y cuando combinamos las dos cosas así somos sentipensantes”. Entrevista fue realizada por Rafael Bassi Labarrera y David Britton para el documental José Barros, Rey De Reyes, en el año 2007.

Ver https://www.youtube.com/watch?v=LbJWqetRuMo

Estudios de la Paz y el Conflicto, Revista Latinoamericana, IUDPAS-UNAH, Número Especial 1, 2019, pp. 111-119.

DOI: $10.5377 /$ rlpc.v0i0.9507
} 
Fals Borda, O. (2009). Una sociología sentipensante para América Latina. Orlando Fals Borda Antología. En Moncayo, V.M. (Comp.). Bogotá: Siglo del Hombre Editores CLACSO.

Gentile, B. (2013). Cadáveres y Votos. Claves para pensar la Violencia Institucional. Buenos Aires: Editorial de Autores de Argentina.

Lenkersdorf C. (2008). “Aprender a Escuchar. Enseñanzas Maya-tojolabales”. México: Plaza y Valdés.

Martín-Baró, I. (1989). Psicología Social de la liberación. Madrid: Trotta.

Quintar, E. (2006). La enseñanza como puente a la vida. México: Instituto Politécnico Nacional.

Soussa, B. (2018). Epistemologías del Sur. Buenos Aires: Centro de Estudios Sociales.

Zemelman, H. (2002). Necesidad de Conciencia, Un modo de construir conocimiento. Barcelona: Anthropos.

\section{REFERENCIAS DE LAS AUTORAS}

Dennis Dussán Márquez, Socióloga. Activista por la Paz. Excombatiente de la insurgencia del proceso de Paz entre el Gobierno Nacional y la Corriente de Renovación Socialista el 9 de abril de 1994. Integrante de la Red Nacional de Mujeres Excombatientes de la Insurgencia. Docente de la Universidad de la Amazonia. Coordinadora del Grupo de Investigación Mandala de Pensamiento y del semillero Inti-Wayra. Magister y Doctora en Pensamiento y Cultura para América Latina del Instituto de Pensamiento y Cultura en América Latina (Ipecal-México). dennisdussan@yahoo.es ORCID: 0000-0003-2606$278 \mathrm{X}$

Luisa Fernanda Toro Cruz, Estudiante de séptimo semestre de Derecho de la Universidad de la Amazonia. Activista en Construcción de paz. Coordinadora estudiantil del semillero Inti-Wayra adscrito al programa de Derecho y a la oficina de paz de la Universidad. Integrante del grupo de investigación Mándala De Pensamiento. lufertoro17@hotmail.com ORCID: 0000-0002-7506-8654

Recibido: 12-10-2019

Aceptado: 04-02-2020

(c) (i) Licencia Creative Commons Reconocimiento 4.0 (CC BY 4.0). 